\title{
Flexible and tandem quantum-dot light-emitting diodes with individually addressable red/green/blue emission
}

\author{
Qiang Su${ }^{1}$, Heng Zhang ${ }^{1}$ and Shuming Chen $\mathbb{D}^{1,2 \otimes}$
}

Tandem quantum-dot light-emitting diodes (QLEDs) with multiple QLED elements vertically connected by the intermediate electrodes offer the advantages of high brightness and long lifetime. However, it is challenging to individually address each QLED element in conventional tandem structures. To address this challenge, here, transparent QLEDs built on flexible plastic substrates are developed as the building blocks for the tandem QLEDs. By vertically integrating a red, a green, and a blue transparent QLEDs with an ultraviolet glue, the resultant tandem QLED can emit separately controllable red/green/blue (R/G/B) emission with an external quantum efficiency (EQE) of $12.0 \% / 8.5 \% / 4.5 \%$, respectively. Enabled by the transparent and extractable IZO electrodes, the QLED elements can also be connected in series or in parallel with an EQE of $24.8 \%$ or $8.2 \%$, respectively. Our work provides a new implementation strategy for the realization of tandem QLEDs with individually addressable R/G/B emission for both display and lighting applications.

npj Flexible Electronics (2021)5:8; https://doi.org/10.1038/s41528-021-00106-y

\section{INTRODUCTION}

Tandem quantum-dot (QD) light-emitting diodes (LEDs) with multiple LED elements vertically stacked offer the advantages of high brightness and long lifetime, and thus have been hotly investigated as one of the promising displays and lighting devices $^{1-4}$. In conventional tandem QLEDs, the LED elements are connected by the intermediate connection layers (ICL), which typically consist of a p-type and a n-type semiconductor layers and have a function of generating charge carriers for adjacent LED elements $^{5-7}$. In conventional tandem structures, the LED elements can only be serially connected and thus they are simultaneously turned on, when a voltage is applied to the terminals of the devices $^{1-4,8-10}$. At a certain driving current, the brightness of a tandem LED is $n$-fold higher than that of a single device, making it particularly favorable for application scenarios, where high brightness is a must, such as lighting, large area display, and phototherapy ${ }^{11-15}$.

If the LED elements can be individually addressed, the tandem LED can offer more functionalities other than high brightness. For example, by separately controlling the emission of the red/green/ blue (R/G/B) LED elements, a single-tandem LED can serve as a full-color pixel for displays. Compared to the conventional fullcolor displays with horizontal R/G/B side-by-side pixel arrangement, the vertical $R / G / B$ pixel configuration can significantly improve the resolution and the aperture ratio of a display ${ }^{16-20}$. In addition, a single-tandem LED can emit arbitrary or tunable colors by vertically and spatially mixing the emission of the R/G/B LED elements. Furthermore, the LED elements can be connected in different modes, such as in series or in parallel, thus enabling the tandem LED to display a high brightness either at a lower current or at a smaller voltage.

Although tandem LED with individually addressed emission offers many advantages, it is challenging to develop such a kind of structure. Firstly, the ICL used to connect the LED elements should be transparent and conductive. Secondly, the ICL should be patterned and extracted to work as an independent electrode.
Finally, the entire fabrication process should be damage free to all functional layers. We have recently demonstrated a tandem LED with individually addressable blue and yellow emission by stacking a yellow QLED and a blue organic LED, using an indium zinc oxide (IZO) transparent and conductive electrode ${ }^{21}$. Very recently, Sun et al. have developed a tandem LED with separately controllable R/G/B emission by using a patterned AgNWs/resin/ AgNWs as the intermediate addressing electrode ${ }^{20}$. However, the performance of the resultant tandem LED is moderate, since it is difficult to achieve a highly transparent intermediate electrode and a damage-free solution fabrication process especially when tens of functional layers are involved. Moreover, it seems impossible to connect the intermediate electrodes with the electrodes of the bottom driving thin-film transistors (TFTs), making the developed structures useless for display applications.

In this work, we attempt to address the aforementioned challenges by developing the transparent QLEDs as the building blocks for constructing damage-free tandem QLEDs with individually addressable emission. The R/G/B transparent QLEDs are independently built on the flexible polyethylene naphthalate (PEN) substrates, and then they are vertically assembled by using an ultraviolet (UV)-curable glue; by extracting the electrodes of each LED elements, the resultant tandem QLED can emit separately controllable $\mathrm{R} / \mathrm{G} / \mathrm{B}$ emission with an external quantum efficiency (EQE) of $12.0 \% / 8.5 \% / 4.5 \%$, respectively. Since each LED element is independently fabricated on its own substrate, the solvent damage during fabrication of the top LED elements is eliminated, consequently enabling the LED elements to exhibit a high efficiency. Moreover, it is easy to realize a high-resolution monochromic display by integrating the driving TFT with each LED element, and by stacking a red, a green, and a blue transparent display, a high-resolution, color-patterning-free and full-color display can be realized. Our work provides a new implementation strategy for the realization of tandem QLEDs with individually addressable R/G/B emission for both display and lighting applications.

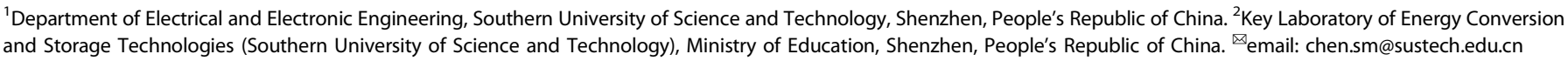




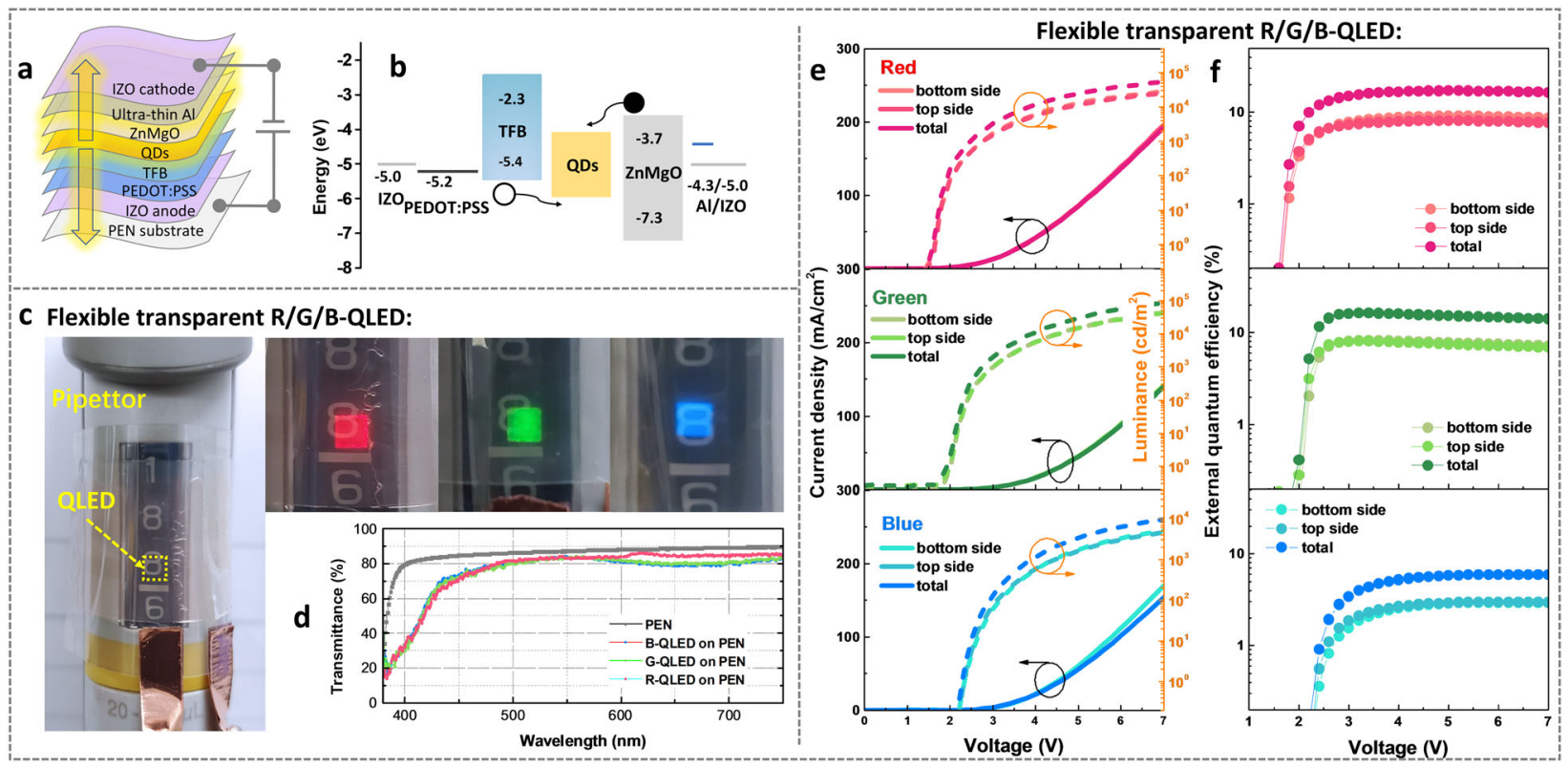

Fig. 1 Flexible and transparent R/G/B-QLED. a Schematic device structure and $\mathbf{b}$ energy levels of the functional layers of the transparent devices. c Photographs of the flexible and transparent R/G/B-QLED attached to the pipettor no matter what the devices were on or off, the numeral below the devices can be clearly recognized, demonstrating a excellent transparency and flexibility. $\mathbf{d}$ The transmittance of PEN and R/G/B-QLED. e The current density-voltage-luminance $(J-V-L)$ and $\mathbf{f}$ the external quantum efficiency-voltage (EQE- $V$ ) characteristics of R/G/B-QLED.

\section{RESULTS AND DISCUSSION}

\section{Flexible and transparent QLEDs}

Transparent QLED with the structure of IZO/(180 nm)/PEDOT:PSS/ $\mathrm{TFB} / \mathrm{QDs} / \mathrm{ZnMgO} / \mathrm{Al}(2 \mathrm{~nm}) / \mathrm{IZO}(80 \mathrm{~nm})$ was fabricated on a thin $(50 \mu \mathrm{m})$ PEN substrate, where the $2 \mathrm{~nm}$ Al deposited by evaporation serves as a buffer layer to protect the bottom functional layers from ion bombardment damage during sputter deposition of the top IZO cathode ${ }^{21,22}$. IZO was employed as bottom anode and top cathode because of its high transparency, high conductivity, and small bombardment damage to the underneath PEN substrate or functional layers. The schematic device structure and the energy levels (taken from refs. ${ }^{14,21,23,24}$ ) of the functional layers of the transparent QLED are shown in Fig. 1a, b. Thanks to the high transparency of IZO electrodes and PEN substrates, the resultant devices exhibit a high transparency of $\sim 80 \%$ (Fig. 1d), which is important for realizing efficient tandem devices, as will be discussed later. The transparency and flexibility of the resultant devices are demonstrated in Fig. 1c and Supplementary movie 1, where the devices were bent and attached to a cylindrical surface of a pipette. The devices can work normally in the bending state and the numeral below the devices can be clearly recognized no matter what the devices were on or off, demonstrating a excellent transparency and flexibility. As shown in Fig. 1f, the red, green, and blue transparent QLEDs exhibit a total EQE (including bottom and top sides) of $17.2 \%, 16.3 \%$, and $6.0 \%$, respectively, which is very close to those of the regular bottom QLEDs (Supplementary Fig. 1), indicating that the sputtered IZO top cathode does not affect the performance of the transparent devices. The corresponding power efficiency is shown in Supplementary Fig. 2.

\section{Flexible tandem QLEDs}

With the transparent and efficient red, green, and blue QLEDs as the building blocks, we can then construct a tandem LED by vertically assembling the devices. Figure $2 a, b$ shows the schematic structure and the assembling processes of the tandem LED. A UV-curable resin that can directly be spun-cast onto the devices was used to encapsulate the LEDs and bond the LED elements. By integrating a transparent blue, a transparent green, and a regular bottom-emitting red LED element using the UV glue, a bottom-emitting tandem LED can be realized. Compared to the demonstrated methods ${ }^{1-4,20}$, the advantages of our design including: (1) the red, green, and blue emission can be individually addressed by extracting the electrodes of each LED element; (2) the issue of solvent damage during the deposition of tens of functional layers in conventional tandem LEDs can be avoided since each LED element is independently built on its own substrate; (3) the resultant tandem LED could be used for display applications; by equipping each colored QLED with the driving TFTs on the same substrate, a monochromic display can be easily realized, and by integrating a red, a green, and a blue display, a full-color display with vertical R/G/B pixel configuration can be achieved; consequently, the problematic color-pattering process can be eliminated and the resolution, the aperture ratio can be significantly improved as compared to conventional displays with patterned side-by-side R/G/B pixels, as schematically illustrated in Fig. 2c; (4) the LED elements can be connected either in conventional series mode or in parallel mode, and thereby can enable the tandem LED to exhibit a high brightness either at a very low current or at a very small voltage, depending on the requirement of practical applications, as shown in Fig. 2 d.

As an example, we demonstrate the capability of individually addressing the emission of each LED element. As shown in Fig. 2e, the red, green, and blue saturated emission can be separately controlled by only applying the driving signals to the specific LED element. Also, tunable colors such as yellow, pink, cyan, and white can be easily achieved by mixing the emission of two or three LED elements. Theoretically, the colors can be tuned in a wide range, such as from red to blue, from red to green, or from green to blue, and also, arbitrary colors that are within the color triangle defined by the LED elements can be generated, as shown in Fig. $2 \mathrm{f}$ and demonstrated in Supplementary movie 2. Enabled by the individually addressable R/G/B emission, the color, the brightness, and the color temperature of the emission can all be independently tuned by simply varying the driving signals, making the resultant tandem LED very attractive for tunable lighting application. 


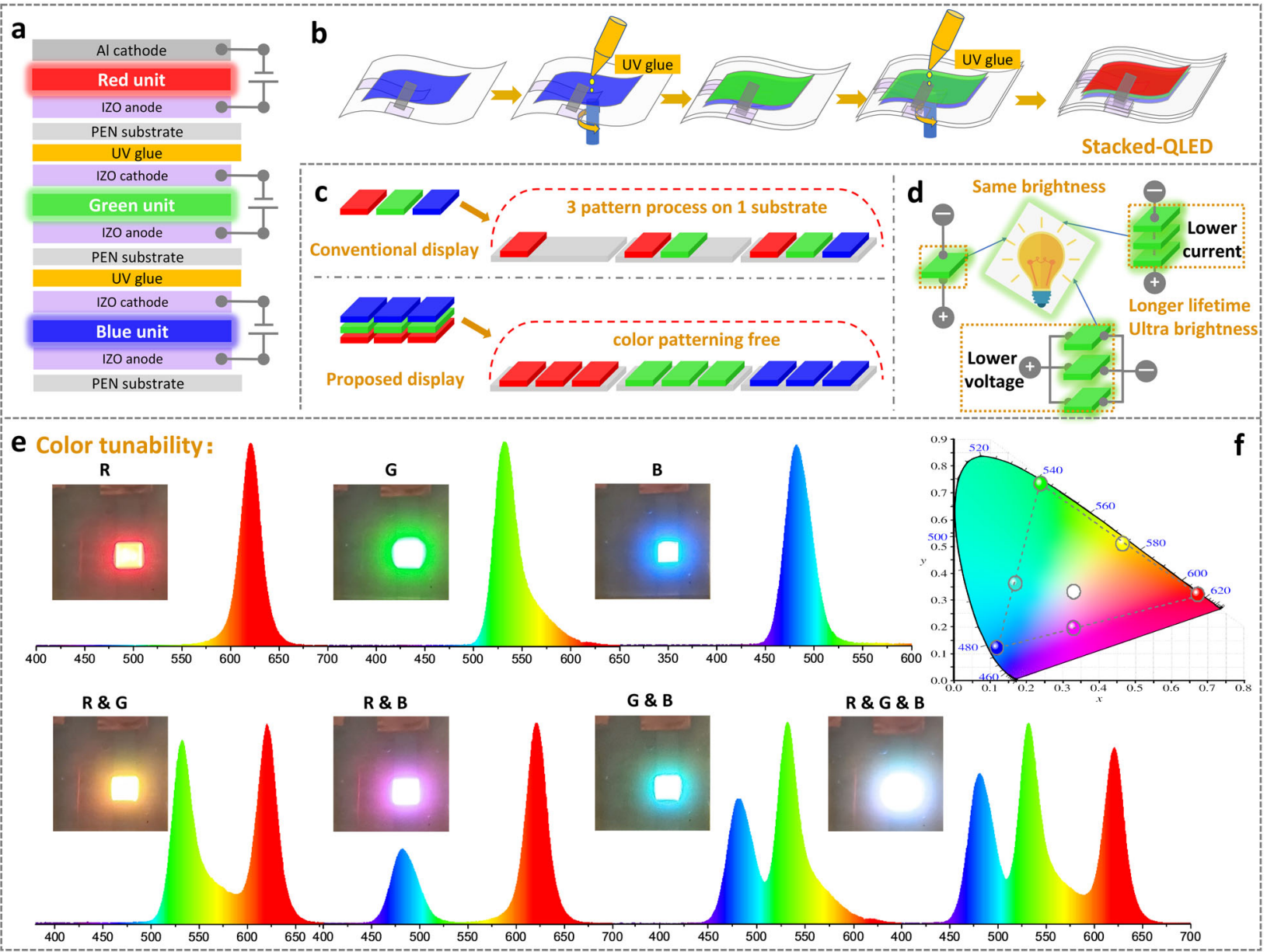

Fig. 2 Tandem QLED with individually addressable R/G/B emission. a The schematic device structure and $\mathbf{b}$ assembling processes of the tandem LED. c Comparison of a conventional full-color display with patterned horizontal R/G/B side-by-side pixel arrangement, and our proposed display with the color-patterning-free vertical R/G/B pixel configuration. Compared to conventional scheme, our method can significantly improve the resolution and the aperture ratio of a display. $\mathbf{d}$ The LED elements can be connected either in series or in parallel mode, and thus our tandem LED can display a high brightness either at a very low current or at a very small voltage, which is beneficial to their lifetime. e Photos and spectra of the tandem QLEDs demonstrating that the R/G/B emission can be separately controlled and tunable colors can also be achieved by varying the driving signals. $f$ The color coordinates of the achievable colors by the tandem QLEDs.

The performances of the red, green, and blue emission in the tandem structure are then evaluated. As shown in Fig. 3a, b, by assembling the red, green, and blue LED elements as a tandem device, their EQEs are reduced from $17.2 \% / 16.3 \% / 6.0 \%$ to $12.0 \% /$ $8.5 \% / 4.5 \%$, which are reduced by $30 \% / 48 \% / 25 \%$, for $\mathrm{R} / \mathrm{G} / \mathrm{B}$ emission, respectively. The corresponding power efficiency is shown in Supplementary Fig. 2. The reason for the reduced emission is mainly ascribed to the absorption of the devices. As shown in Fig. 1d and Supplementary Fig. 3, a single LED exhibits a transmittance of $\sim 80 \%$, whereas a stack of two LED elements shows a transmittance of $65-70 \%$. By placing the red LED on the very top side, its emission is attenuated when passing through the green and the blue LED, and consequently, only $65-70 \%$ of its emission can be output, as schematically shown in Fig. $3 \mathrm{~g}$. Similarly, the emission of the green and the blue are attenuated when passing through their partners. Therefore, to reduce the absorption losses and improve the efficiency, the transparency of the LED elements should be further improved, which could be realized by using more transparent electrodes and substrates.

Enabled by the individually addressable emission, the LED elements can be connected either in series or in parallel mode. As shown in Fig. 3c, d, under parallel connection, all LED elements are driven by the same voltage and the resultant tandem LED exhibits a very high brightness of $130,654 \mathrm{~cd} \mathrm{~m}^{-2}$ at $7 \mathrm{~V}$, which is approximately equal to the sum of those of the LED elements. Whereas under series connection, all LED elements share the same current, and consequently the resultant tandem LED displays a high EQE of $24.8 \%$, as shown in Fig. 3e, $\mathrm{f}$. Depending on the requirement of practical applications, the tandem LED can be operated at a high brightness of $>100,000 \mathrm{~cd} \mathrm{~m}^{-2}$ either at a small voltage of $6.3 \mathrm{~V}$ or a low current of $150 \mathrm{~mA} \mathrm{~cm}^{-2}$, which is beneficial to the improvement of their lifetime.

In summary, by employing magnetron sputtered IZO as the transparent electrode, we have demonstrated flexible, transparent R/G/B QLEDs with the EQEs of $17.2 \% / 16.3 \% / 6.0 \%$, respectively, accompanying with a high transparency of $\sim 80 \%$. By using the transparent QLEDs as the building blocks, tandem QLED with individually addressable emission has been realized. The developed method eliminates the issue of solvent damage in conventional tandem devices and enables us to separately control the emission of each LED element. The demonstrated tandem QLED could be used for full-color displays, which could eliminate the problematic color-patterning processes, and could significantly improve the resolution and the aperture ratio of the displays; meanwhile, with the merits of tunable emission and high brightness either at small voltage or low current, the demonstrated tandem QLED could be attractive for lighting applications. 

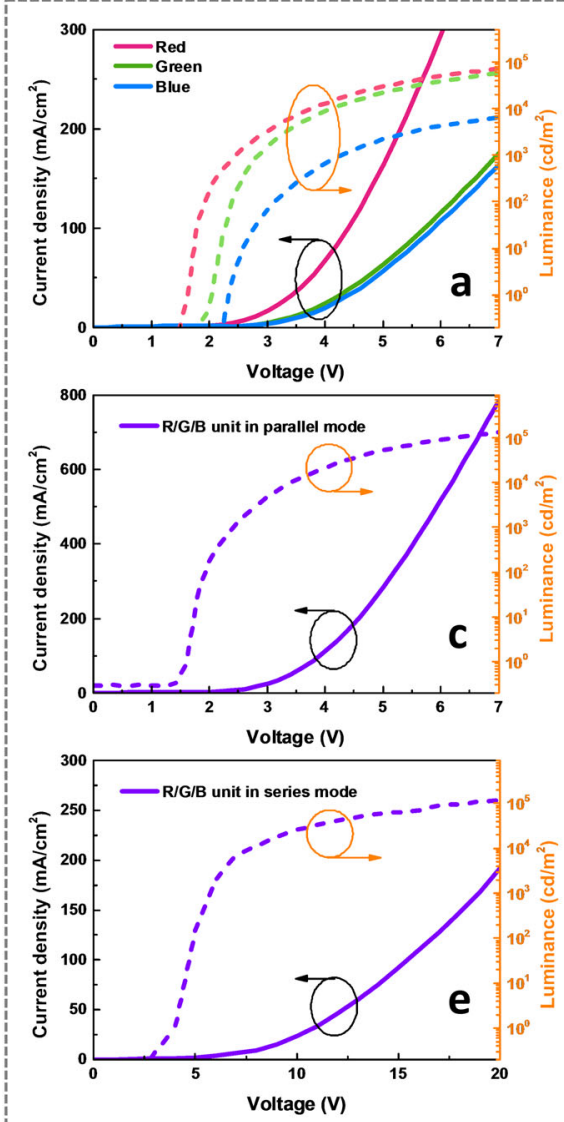
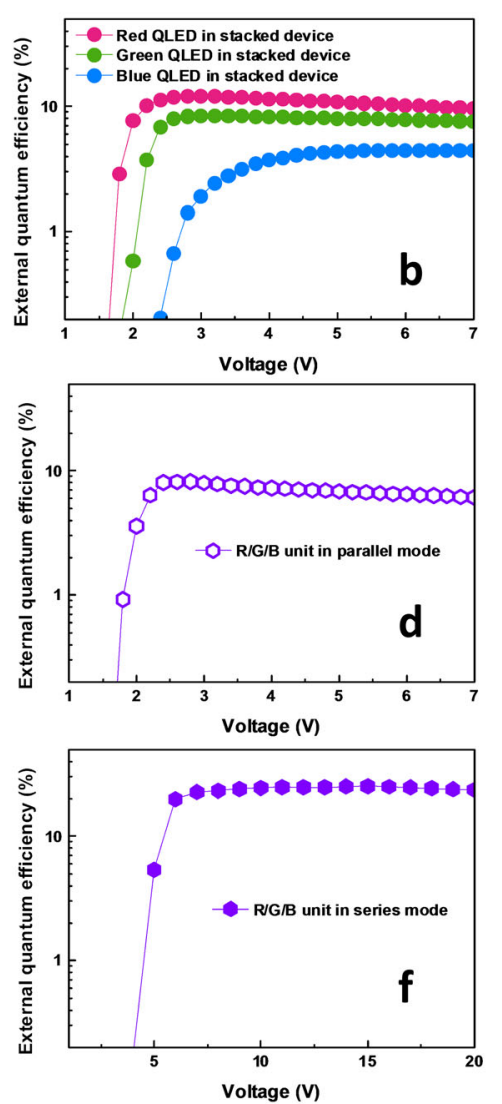

g Luminance loss of R/G/B-QLED in stacked device

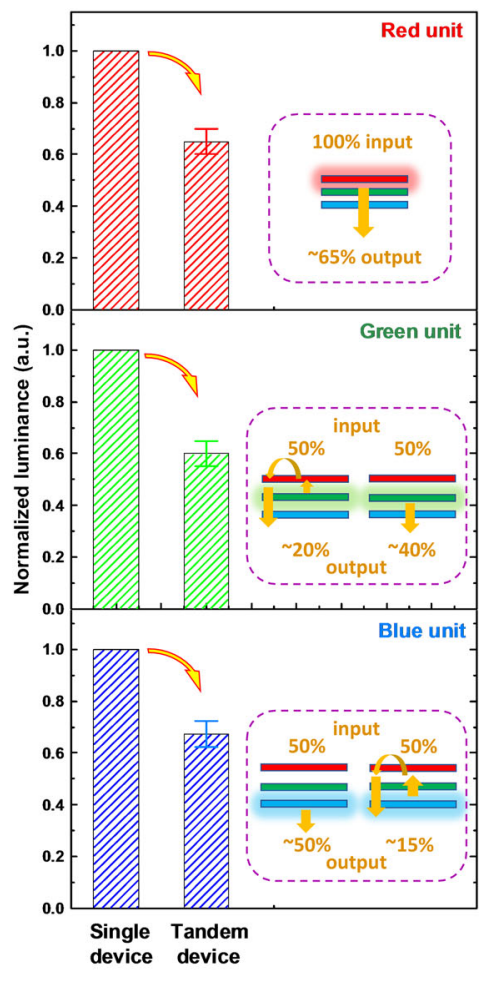

Fig. 3 Performance of the tandem QLED. $\mathbf{a}$ The $J-V-L$ and $\mathbf{b}$ the EQE- $V$ characteristics of the R/G/B emission in the tandem structure. The $J-$ $V-L$ and the EQE- $V$ characteristics of the tandem LED operated at $\mathbf{c}$, $\mathbf{d}$ parallel and $\mathbf{e}$, $\mathbf{f}$ series connection mode, respectively. $\mathbf{g}$ Analysis of the luminance losses of the R/G/B emission in the tandem structure. Because of the absorption of the devices, the emission is attenuated when passing through each LED element. The error bar here is $\pm 5 \%$.

\section{METHODS}

\section{Device structures}

Transparent QLEDs with the structure of PEN/IZO $(180 \mathrm{~nm}) / \mathrm{PEDOT}$ :PSS $(45 \mathrm{~nm}) / \mathrm{TFB}(40 \mathrm{~nm}) / \mathrm{QDs}(\sim 15 \mathrm{~nm}) / \mathrm{ZnMgO}(40 \mathrm{~nm}) / \mathrm{Al}(2 \mathrm{~nm}) / \mathrm{IZO}(80 \mathrm{~nm})$ were fabricated on a thin $(50 \mu \mathrm{m})$ PEN substrate, where the $2 \mathrm{~nm} \mathrm{Al}$ deposited by evaporation serves as a buffer layer to protect the bottom functional layers from ion bombardment damage, during sputter deposition of the top IZO cathode.

Tandem QLEDs with the structure of PEN/IZO/PEDOT:PSS/TFB/blue QDs/ZnMgO/Al (2 nm)/IZO/UV glue/PEN/IZO/PEDOT:PSS/TFB/green QDs/ ZnMgO/Al (2 nm)//ZO/UV glue/PEN//ZO/PEDOT: PSS/TFB/red QDs/ZnMgO/ Al $(100 \mathrm{~nm})$ were fabricated by bonding with UV glue.

IZO, PEDOT:PSS (poly (ethylenedioxythiophene)/polystyrenesulfonate), TFB (poly[(9,9-dioctylfluorenyl-2,7-diyl)-co-(4,4'-(N-(pbutylphenyl))diphenylamine)]), QDs, and ZnMgO work as anode/cathode, hole injection layer (HIL), hole transport layer (HTL), light emission layer (EML), and electron transport layer $(E T L)$, respectively.

\section{Device fabrication}

Transparent QLED. First, the cleaned PEN plastic substrates were transferred to a magnetron sputtering system to deposit the IZO anode $\left(180 \mathrm{~nm}, \sim 30 \Omega \mathrm{sq}^{-1}\right)$ at a working pressure of $4.5 \times 10^{-1} \mathrm{~Pa}$, a power of $100 \mathrm{~W}$, an Ar flow of $20 \mathrm{sccm}$. The anode was patterned by using a shadow mask. The IZO target used here is composed of $90 \mathrm{wt} \% \ln _{2} \mathrm{O}_{3}$ and $10 \mathrm{wt} \%$ $\mathrm{ZnO}$. Then, these flexible PEN/IZO substrates were bound to glass substrates for subsequent processing. The HIL was formed by spincasting PEDOT:PSS (Clevios Al 4083) at 3000 r.p.m. and baked at $120^{\circ} \mathrm{C}$ for $20 \mathrm{~min}$ in the atmosphere after treating the IZO with $\mathrm{O}_{2}$ plasma for 6 min. After that, the samples were transferred into an $\mathrm{N}_{2}$-filled glovebox to prepare the subsequent functional layers. The TFB HTL was deposited by spin-casting its solution ( $8 \mathrm{mg} \mathrm{mL}^{-1}$ in chlorobenzene) at 3000 r.p.m. for
$45 \mathrm{~s}$ and baked at $100^{\circ} \mathrm{C}$ for $20 \mathrm{~min}$. Then, the QDs EMLs were obtained by spin-casting the QDs solution at 3000 r.p.m., and baked at $100^{\circ} \mathrm{C}$ for $5 \mathrm{~min}$. The R/G/B QDs (CdSe/ZnS) were dissolved in $n$-octane with a concentration of 15,10 , and $10 \mathrm{mg} \mathrm{mL}^{-1}$, respectively. Afterward, $\mathrm{ZnMgO}$ nanoparticles (20 mg mL ${ }^{-1}$ in ethanol) were spin-coated on QDs films at 2500 r.p.m. and annealed at $80^{\circ} \mathrm{C}$ for $20 \mathrm{~min}$. Subsequently, the samples were transferred to a high-vacuum evaporation chamber to deposit a $2 \mathrm{~nm}$ Al buffer layer with an evaporation rate of $1.5 \AA \mathrm{s}^{-1}$ at a base pressure of $5 \times 10^{-4}$ Pa. Finally, the IZO cathode with a thickness of $80 \mathrm{~nm}\left(\sim 80 \Omega \mathrm{sq}^{-1}\right)$ was fabricated by magnetron sputtering at a working pressure of $4.5 \times 10^{-1} \mathrm{~Pa}$, a power of $50 \mathrm{~W}$, an Ar flow of $20 \mathrm{sccm}$. The Al cathode was prepared in the highvacuum evaporation chamber with an evaporation rate of $5 \AA \mathrm{s}^{-1}$ at a base pressure of $5 \times 10^{-4} \mathrm{~Pa}$ after completing the ZnMgO ETL. The QDs and $\mathrm{ZnMgO}$ nanoparticles were purchased from Suzhou Xingshuo Nanotech Co., Ltd and Guangdong Poly OptoElectronics Co., Ltd, respectively.

Tandem QLED. A UV-curable resin (Loctite 3311, viscosity: 300) was used to encapsulate the transparent QLED and bond the QLED elements. The UV-resin was directly spun-cast on the first QLED element at 3000 r.p.m., and then the second QLED was placed on top of the UV-resin and was aligned with the first QLED, followed by curing the resin by a UV lamp ( $36 \mathrm{w}$ ) for $8 \mathrm{~min}$. By repeating the above processes, tandem QLED with the red, green, and blue QLED elements vertically assembled was obtained.

\section{Device characterization}

The thicknesses of the functional layers and IZO electrodes were measured using a Bruker DektakXT stylus profiler. The evaporation rates and the thicknesses of thin Al, Al cathode, were in situ monitored by a quartz crystal microbalance. The sheet resistance of IZO electrodes was measured by a four-point probe resistivity measurement system. The electroluminescence (EL) spectra of QLEDs were measured by a PR 670 spectrometer, and 
the $J-V-L$ characteristics of QLEDs were assessed by using a dual-channel Keithley 2614B programmable source meter with a PIN-25D calibrated silicon photodiode under ambient conditions.

Measurement of EQE was achieved by a method recommended by SR Forrest ${ }^{25}$, in which a large area PIN-25D silicon photodiode (made by OSI Optoelectronics) was placed in close contact with the devices. The active area $(2 \mathrm{~mm} \times 2 \mathrm{~mm})$ of the device under test is much smaller than that of the photodiode $\left(613 \mathrm{~mm}^{2}\right)$ so that all light emitted by the device can be detected by the photodiode. The setup was placed in a home-made black box so that only the light emitted by the QLED surface facing to the photodiode is detected, while other emission (such as the top-side emission of the transparent QLED) was absorbed by the black box. The driving current through the devices was monitored simultaneously by a dualchannel Keithley 2614B source measure unit. Prior to the measurement of EQE and $J-V-L$ characteristics, the EL spectra of the QLED were measured by a PR 670 spectrometer. By inputting the emission area $\left(4 \mathrm{~mm}^{2}\right)$, the emission solid angle $(\pi)$, and the EL spectra to a home-made software, the EQE and the luminance can be calculated ${ }^{25}$. The luminance acquired from this method was calibrated by a PR 670 spectrometer, which can measure the luminance directly.

For the devices with the bias-dependent spectra (such as our tandem QLEDs operated in series mode), their spectra at different voltage were first measured and then input to the software; then the EQE and the luminance at each voltage can be calculated by using the input bias-dependent spectra. For the tandem QLED operated in parallel mode, the EQE and the $J-V-L$ of each QLED element were independently measured, and then the EQE of the tandem device can be calculated by using the below Eq. (1):

$$
\mathrm{EQE}_{\text {parallel }}(V)=\frac{\operatorname{EQE}_{\text {Red }}(V) \times J_{\text {Red }}(V)+\mathrm{EQE}_{\text {Green }}(V) \times J_{\text {Green }}(V)+\operatorname{EQEE}_{\text {Blue }}(V) \times J_{\text {Blue }}(V)}{J_{\text {Red }}(V)+J_{\text {Green }}(V)+J_{\text {Blue }}(V)}
$$

where EQE $(V)$ and $J(V)$ are the external quantum efficiency and the current density of each QLED element operated at a specific driving voltage $V$, respectively.

\section{DATA AVAILABILITY}

The data that support the findings of this study are available from the corresponding author upon reasonable request.

Received: 12 December 2020; Accepted: 8 March 2021; Published online: 26 March 2021

\section{REFERENCES}

1. Kim, H. M., Lee, J., Hwang, E., Kim, J. \& Jang, J. Inverted tandem architecture of quantum-dot light emitting diodes with solution processed charge generation layers. Dig. Tech. Pap. Soc. Inf. Disp. Int. Symp. 47, 1480-1483 (2016).

2. Zhang, H., Chen, S. M. \& Sun, X. W. Efficient red/green/blue tandem quantum-dot light-emitting diodes with external quantum efficiency exceeding $21 \%$. ACS Nano 12, 697-704 (2018)

3. Jiang, C. B. et al. Fully solution-processed tandem white quantum-dot lightemitting diode with an external quantum efficiency exceeding $25 \%$. ACS Nano 12, 6040-6049 (2018).

4. Cao, F. et al. High-efficiency, solution-processed white quantum dot light-emitting diodes with serially stacked red/green/blue units. Adv. Opt. Mater. 6, 1800652 (2018)

5. Han, C.-Y. \& Yang, H. Development of colloidal quantum dots for electrically driven light-emitting devices. J. Korean Ceram. Soc. 54, 449-469 (2017).

6. Zhang, H., Sun, X. W. \& Chen, S. M. Over $100 \mathrm{~cd} \mathrm{~A}^{-1}$ efficient quantum dot lightemitting diodes with inverted tandem structure. Adv. Funct. Mater. 27, 1700610 (2017).

7. Chiba, T., Pu, Y.-J. \& Kido, J. in Handbook of Organic Light-Emitting Diodes (eds Chihaya A. Reiji H., Hironori K. \& Takatoshi T.) 1-22 (Springer, 2018).

8. Hofle, S. et al. Solution processed, white emitting tandem organic light-emitting diodes with inverted device architecture. Adv. Mater. 26, 5155-5159 (2014).

9. $\mathrm{Pu}, \mathrm{Y}$.-J. et al. Fabrication of organic light-emitting devices comprising stacked lightemitting units by solution-based processes. Adv. Mater. 27, 1327-1332 (2015).

10. Fung, M. K., Li, Y. Q. \& Liao, L. S. Tandem organic light-emitting diodes. Adv. Mater. 28, 10381-10408 (2016).

11. Supran, G. J. et al. QLEDs for displays and solid-state lighting. MRS Bull. 38 703-711 (2013).

12. Dai, X. L., Deng, Y. Z., Peng, X. G. \& Jin, Y. Z. Quantum-dot light-emitting diodes for large-area displays: towards the dawn of commercialization. Adv. Mater. 29, 1607022 (2017).
13. Lim, J., Park, Y. S., Wu, K. F., Yun, H. J. \& Klimov, V. I. Droop-free colloidal quantum dot light-emitting diodes. Nano Lett. 18, 6645-6653 (2018).

14. Shen, H. B. et al. Visible quantum dot light-emitting diodes with simultaneous high brightness and efficiency. Nat. Photonics 13, 192-197 (2019).

15. Chen, H. et al. Quantum dot light emitting devices for photomedical applications. J. Soc. Inf. Disp. 25, 177-184 (2017).

16. Forrest, S. R. et al. The stacked OLED (SOLED): a new type of organic device for achieving high-resolution full-color displays. Synth. Met. 91, 9-13 (1997).

17. Hui, K. N., Wang, X. H., Li, Z. L., Lai, P. T. \& Choi, H. W. Design of Vertically-stacked polychromatic light-emitting diodes. Opt. Express 17, 9873-9878 (2009).

18. Kang, C. M. et al. Fabrication of a vertically-stacked passive-matrix micro-LED array structure for a dual color display. Opt. Express 25, 2489-2495 (2017).

19. Geum, D. M. et al. Strategy toward the fabrication of ultrahigh-resolution microLED displays by bonding-interface-engineered vertical stacking and surface passivation. Nanoscale 11, 23139-23148 (2019).

20. Sun, J. et al. Large-area tunable red/green/blue tri-stacked quantum dot lightemitting diode using sandwich-structured transparent silver nanowires electrodes. ACS Appl. Mater. Interfaces 12, 48820-48827 (2020).

21. Zhang, H., Su, Q. \& Chen, S. M. Quantum-dot and organic hybrid tandem lightemitting diodes with multi-functionality of full-color-tunability and white-lightemission. Nat. Commun. 11, 2826 (2020).

22. Choi, M. K. et al. Extremely vivid, highly transparent, and ultrathin quantum dot light-emitting diodes. Adv. Mater. 30, 1703279 (2018).

23. Dai, X. L. et al. Solution-processed, high-performance light-emitting diodes based on quantum dots. Nature 515, 96-99 (2014).

24. Sun, Y. Z. et al. Efficient quantum dot light-emitting diodes with a $\mathrm{Zn}_{0.85} \mathrm{Mg}_{0.15} \mathrm{O}$ interfacial modification layer. Nanoscale 9, 8962-8969 (2017).

25. Forrest, S. R., Bradley, D. D. \& Thompson, M. E. Measuring the Efficiency of Organic Light-Emitting Devices. Adv. Mater. 15, 1043-1048 (2003).

\section{ACKNOWLEDGEMENTS}

This work was supported by the National Natural Science Foundation of China (61775090) and the Guangdong Natural Science Funds for Distinguished Young Scholars (2016A030306017).

\section{AUTHOR CONTRIBUTIONS}

S.C. conceived the idea, supervised the device fabrication, data collection and wrote the paper. Q.S. conducted the experiments and drew the figures. S.C., Q.S. and H.Z discussed the results and commented on the manuscript.

\section{COMPETING INTERESTS}

The authors declare no competing interests.

\section{ADDITIONAL INFORMATION}

Supplementary information The online version contains supplementary material available at https://doi.org/10.1038/s41528-021-00106-y.

Correspondence and requests for materials should be addressed to S.C.

Reprints and permission information is available at http://www.nature.com/reprints

Publisher's note Springer Nature remains neutral with regard to jurisdictional claims in published maps and institutional affiliations.

\footnotetext{
Open Access This article is licensed under a Creative Commons Attribution 4.0 International License, which permits use, sharing, adaptation, distribution and reproduction in any medium or format, as long as you give
appropriate credit to the original author(s) and the source, provide a link to the Creative Commons license, and indicate if changes were made. The images or other third party material in this article are included in the article's Creative Commons license, unless indicated otherwise in a credit line to the material. If material is not included in the article's Creative Commons license and your intended use is not permitted by statutory regulation or exceeds the permitted use, you will need to obtain permission directly from the copyright holder. To view a copy of this license, visit http://creativecommons. org/licenses/by/4.0/.
}

(c) The Author(s) 2021 